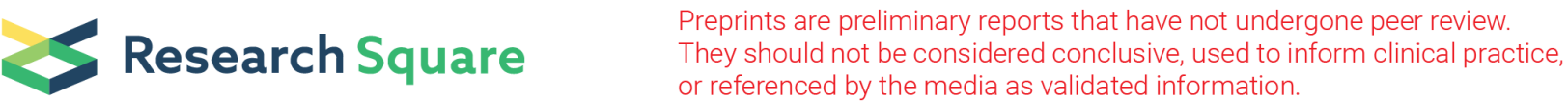

\section{The feasibility of anterior transpedicle screw fixation in lumbosacral spine: a radiographic and cadaveric study}

\section{Wei-Xing Xu}

Tongde Hospital Of Zhejiang Province

Bin Xu ( $\nabla$ spotxu@163.com )

Tongde Hospital Of Zhejiang Province https://orcid.org/0000-0002-8614-953X

\section{Wei-Guo Ding}

Tongde Hospital Of Zhejiang Province

\section{Hong-Feng Sheng}

Tongde Hospital Of Zhejiang Province

Di Lu

Tongde Hospital Of Zhejiang Province

\section{Tian-Hong Hu}

Tongde Hospital Of Zhejiang Province

\section{Research article}

Keywords: anterior transpedicle screw, lumbosacral spine, radiographic measurement, safe operating area

Posted Date: November 24th, 2020

DOl: https://doi.org/10.21203/rs.3.rs-50034/v3

License: (c) (1) This work is licensed under a Creative Commons Attribution 4.0 International License. Read Full License 


\section{Abstract}

Background: The anterior transpedicle screw technique for L5 and S1 is crucial for proper anterior lumbar interbody fusion. This study aimed to determine the projection, screw trajectory angle, and bone screw passageway length (BSPL) of the anterior transpedicle screw in L5 and S1, as well as the screw's insertion regularity and the operating area that is safe for its insertion.

Methods: Forty patients with low back pain, all of whom had lumbar computed tomography scans available, were included in a retrospective analysis. Radiographic parameters were measured, including the distances from the projection to the upper endplate, lower endplate, and midline; the transverse and sagittal screws' angles; and the BSPL. Ten fresh adult cadaveric lumbosacral spine segments were chosen to determine the safe anatomical area at which to operate. Finally, anterior transpedicle screws were inserted in L5 and S1 to determine the regularity of anterior pedicle screw insertion.

Results: We measured the anterior projection parameters, including the distances to the upper endplate (L5:12.5 $\pm 1.3 \mathrm{~mm} ; \mathrm{S} 1: 4.54 \pm 0.87 \mathrm{~mm}$ ), lower endplate (L5: $17.3 \pm 1.6 \mathrm{~mm})$, and midline (L5: $6.6 \pm 0.7$ $\mathrm{mm}$; S1: $6.6 \pm 0.6 \mathrm{~mm})$; the screw trajectory angle, including the transverse screw angle (L5: $25.3^{\circ} \pm 2.8^{\circ}$; $\left.\mathrm{S} 1: 25.7^{\circ} \pm 2.6^{\circ}\right)$, sagittal screw angle $\left(\mathrm{L} 5: 17.1^{\circ} \pm 1.7^{\circ} ; \mathrm{S} 1: 22.4^{\circ} \pm 1.1^{\circ}\right)$; and the BSPL (L5: $48.6 \pm 3.5$ $\mathrm{mm} ; \mathrm{S} 1: 48.0 \pm 3.5 \mathrm{~mm}$ ). We then identified the safe operating area and the regularity of L5 and S1 anterior pedicle screw insertions.

Conclusions: We determined the projection, screw trajectory angle, and BSPL of anterior transpedicle screws in L5 and S1, their insertion regularity, and the area in which the operation could be safely performed.

\section{Background}

Lumbar interbody fusion is an effective treatment for spinal disease, including recurrent disc degeneration, spondylolisthesis, degenerative disc disease, pseudarthrosis and spine deformity, spine infection, and tumors [1, 2]. Compared with posterior lumbar interbody fusion, anterior lumbar interbody fusion (ALIF) potentially permits more extensive disc removal, avoids scarring of the neural canal, and preserves the posterior elements [3]. Because biomechanical studies and clinical experience suggest that ALIF with posterior pedicle screw (PPS) augmentation (ALIF-PPS) may provide a superior construct, ALIFPPS is currently used most often. However, ALIF-PPS would increase the number of incision, the operating time and blood loss. While stand-alone ALIF has potential benefit of shorter operating time, less blood loss and avoiding of posterior muscle damage [4, 5].

Hence, numerous studies have designed and applied anterior lumbar internal fixation systems for ALIF. Most of them were vertebral screw-plate fixation systems, whose stability and biomechanical properties were worse than those of the pedicle screw fixation systems [3]. Compared with the vertebral screw-plate fixation system, the pedicle screw fixation system is simple to use and provides good stability for three-column spinal injuries. Thus, augmentation with an anterior pedicle screw (APS) would provide 
better stability for ALIF. Karim, who presented a technique that allows the ALIF procedure to be augmented with APS (ALIF-APS) fixation, found that the stability of the AILF-APS technique is comparable to that provided with ALIF-PPS [3]. Karim, however, did not provide the detailed procedure for APS fixation, including the parameters about the projection, screw trajectory angle, and screw depth.

As two of the most frequently involved segments, L5 and S1 have complex anterior anatomy and unique biomechanics, which pose a clinical challenge for anterior surgical treatment. Because of the complex lumbosacral anatomical structure, various complications are associated with ALIF, including vascular complications, bladder, nerve, ureter, and bowel injures, and abdominal wall problems [6]. With respect to the complex anatomy of the anterior lumbosacral and related surrounding tissue, the concept of a clear space zone (a triangular area on major blood vessels or nerve trunks) in front of the lumbosacral spine emerged [7]. The detailed anatomical parameters of the clear space zone, however, remained unknown.

We therefore conducted this study to obtain radiographic measurements of the anterior transpedicle screw in L5 and S1, which we expected to reveal (1) the position of the anterior pedicle screw projection; (2) the screw's trajectory angle, including the transverse screw and sagittal screw angles; (3) the bone screw passageway length (BSPL); (4) the regularity of anterior pedicle screw insertion; and (5) anatomical parameters of the safe operating area.

\section{Methods}

\section{Specimens}

Ten dry adult cadaveric lumbosacral spine segments were used in this study. Exclusion criteria for the specimens included (1) presence of destructive pathology (tumor, infection, prior surgery, severe degeneration) and (2) severe osteoporotic bone.

\section{Pedicle guide needle placement}

The Ebraheim method [8] was used to determine the pedicle axis and the anterior projection point of L5 and $S 1$. Line $X$ in the transverse plane and line $Y$ in the sagittal plane were drawn on the specimens, as described previously [8]. The two lines intersected on the anterior aspect. This crossing point was determined to be the anterior pedicle axis projection point, which was marked. Guide needles (Kirschner wire) were inserted from the anterior pedicle axis projection point along lines $X$ and $Y$, according to its sagittal and transverse angels. Finally, there were 20 needles being inserted to guide the anterior transpedicle screw in each segments (both side of ten cadavers). C-arm radiography was performed when the front end of the guide needle was at each of four points-anterior projection point, middle of the vertebrae, posterior edge of the vertebrae, the posterior projection point-to ensure accurate positioning of the guide needle. The regularity of the four points, which also means the trajectory of pedicle guide needle was recorded.

\section{Parameter measurements}


Forty patients with low back pain were retrospectively included, each of whom had undergone lumbar CT scanning. None of the patients had lumbar structural damage, malformations, a history of surgery, or lumbosacral transitional vertebrae. Syngo 3D software (Siemens Medical Solutions USA, Inc., Malvern, PA, USA) was used to perform radiographic measurements (Figure 1), including anatomic parameters of anterior projection (distances to upper endplate, lower endplate, and midline), transverse screw angle, sagittal screw angle, and BSPL. Anatomic parameter measurements were performed using calipers and a standard ruler (precision of $1 \mathrm{~mm}$ ) for linear measurements. The safe operating area was calculated in cadavers. Anatomic parameters included the safe operating area (distance from the abdominal aortic bifurcation to the L5 lower edge, distance from the common iliac vein confluence to the L5 lower edge, horizontal distance from the inner edge of the common iliac vein to the L 5 lower edge, distance between S1 holes, L5/S1 vertebral height, and the area in which operating was safe; Figure 2).

\section{Results}

\section{Anterior entry point, screw angle, and BSPL of L5 and S1}

We measured the anterior projection parameters of $L 5$, including the distance to the upper endplate (12.5 $\pm 1.3 \mathrm{~mm})$, the lower endplate $(17.3 \pm 1.6 \mathrm{~mm})$, and the midline $(6.6 \pm 0.7 \mathrm{~mm})$. We also measured the screw trajectory angle, including the transverse screw angle $\left(25.3^{\circ} \pm 2.8^{\circ}\right)$ and sagittal screw angle $\left(17.1^{\circ}\right.$ $\left.\pm 1.7^{\circ}\right)$, as well as the BSPL $(48.6 \pm 3.5 \mathrm{~mm}$; Table 1$)$.

Additionally, we determined the anterior projection parameters for $\mathrm{S} 1$, including the distance to the upper endplate $(4 \mathrm{~mm})$ and the midline $(6.6 \pm 0.6 \mathrm{~mm})$; the screw trajectory angle, including the transverse screw angle $\left(25.7^{\circ} \pm 2.6^{\circ}\right)$ and sagittal screw angle $\left(22.4^{\circ} \pm 1.1^{\circ}\right)$; and the BSPL $(48.0 \pm 3.5 \mathrm{~mm} ;$ Table 1$)$.

\section{Regularity of anterior pedicle screw insertion}

The regularity of L5 anterior pedicle screw insertion is shown in Figure 3 and that of S1 in Figure 4. When the needle is on the anterior projection in the lateral view, it is on the midpoint between the spinous process and the inner edge of the pedicle in the anteroposterior view. When it reaches the posterior vertebral edge in the lateral view, it is on the inner edge of the pedicle in the anteroposterior view. When it reaches the middle of the pedicle in the lateral view, it is on the middle of the pedicle in the anteroposterior view. Finally, when the needle reaches the posterior projection in the lateral view, it is on the outer edge of the pedicle in the anteroposterior view.

\section{Safe operating area}

Our study revealed the anatomical parameters of the safe operating area, including the distance from the abdominal aortic bifurcation to the L5 lower edge $(40.50 \pm 9.40 \mathrm{~mm})$, the distance from the common iliac vein confluence to the L5 lower edge $(27.80 \pm 8.60 \mathrm{~mm})$, and the horizontal distance from the inner edge of the common iliac vein to the L5 lower edge $(37.50 \pm 1.30 \mathrm{~mm})$. The study also determined the distance 
between S1 holes $(29.30 \pm 1.30 \mathrm{~mm})$, the L5/S1 intervertebral height $(17.20 \pm 1.50 \mathrm{~mm})$, and the safe operating area $\left(2058.20 \pm 84.30 \mathrm{~mm}^{2}\right)$.

\section{Discussion}

Since Boucher et al. initially reported use of the posterior transpedicular screw for lumbosacral fusion fixation in 1959 [9], various pedicle screw fixation systems have been used in spinal surgical procedures. They have provided superior postoperative spinal stability and promoted advances in spine surgery [10]. Various studies have researched detailed PPS procedures, including projection parameters, the trajectory angle and depth of the screw [11, 12], and the regularity of pedicle screw insertion [12], each of which is important if PPS is to be performed with high accuracy. Limited studies, however, have reported on the details of procedures using the APS. As an effective means of pedicle screw fixation, APS not only provides superior postoperative spinal stability for ALIF, but allows a shorter operating time, less blood loss, and minimal posterior muscle damage $[4,5]$.

We conducted the present study to determine the anatomic and radiographic parameters of L5 and S1, including projection, the screw's trajectory angle, and the depth of the APS. We found limited information in the literature on the location of the anterior projection, which is important for APS performance. Poor placement of the projection may cause the guide needle to be misplaced and result in complications.

The anterior projection in our study was determined based on the method of Ebraheim [8]. The parameters addressed to determine its location included the distance of the APS to the upper endplate, lower endplate, and midline. The screw trajectory angle was also determined based on the method of Ebraheim [8], and the transverse screw and sagittal screw angles were measured. The screw trajectory angles for APS and PPS should be the same in the same patient. Ebraheim [8] reported that the transverse angle of L5 was $40.6 \pm 2.6$ in men and $39.6 \pm 3.2$ in women, and the sagittal angle of L5 was $2.7 \pm 1.1$ in men and $2.6 \pm 0.9$ in women. To determine the screw trajectory depth, the length of the bone screw passageway was measured. Ebraheim [8] reported that the pedicle length of L5 was $48.3 \pm 2.3 \mathrm{~mm}$ in men and $48.3 \pm 2.4 \mathrm{~mm}$ in women. In the present study, anatomic measurements revealed that the bone screw passageway length was $48.6 \pm 3.5 \mathrm{~mm}$ for $\mathrm{L} 5$ and $48.0 \pm 3.5 \mathrm{~mm}$ for S1.

The BSPL is defined by the length of the screw. Because the vertebra is formed as an irregular cylinder, with the front of vertebral body exhibiting the most anterior border in the lateral view, the needle may perforate the anterolateral cortex, although the lateral view suggests that the needle is still within the vertebra [11]. Therefore, it is important to obtain the ideal needle depth/vertebral width ratio on the lateral view. Weinstein et al. considered the suitable ratio to be $50 \%-80 \%$ [14]. Du et al. suggested that the ratio should be $85 \%-90 \%$ in lumbar vertebrae [15], and Acikbas and Tuncer reported that the suitable ratio was $60 \% \pm 9 \%$ in lumbar vertebrae [16]. Wang et al. found that the ratio was not the same on different lateral projection angle views even if the real length of the needle in the vertebra remained unchanged [11]. They suggested that the suitable ratio of the needle depth/vertebral width ratio on a standard lateral view 
varied from $71.53 \% \pm 5.72 \%$ to $93.28 \% \pm 3.72 \%$ and that the ratio for L5 was $88.20 \% \pm 6.72 \%$. More work is obviously needed to establish a suitable ratio for the APS needle depth.

We also evaluated the regularity of APS insertion in this study, which was guided with high accuracy. A previous study [12] reported that the regularity of PPS insertion was apparent when, progressively, the needle reached the posterior projection in the lateral view, was on the outer edge of the pedicle on the anteroposterior view, reached the middle of the pedicle (lateral view), was on the middle of the pedicle (anteroposterior view), reached the posterior vertebral edge (lateral view), and was on the inner edge of the pedicle (anteroposterior view). Wang et al. reported that the posterior projection of PPS was at the 9 o'clock to 11 o'clock position of the left pedicle and at the 1 o'clock to 3 o'clock position of the right pedicle [11]. This regularity also could be applied to the APS when the needle reaches the posterior projection.

Compared with PPS, APS had higher risk to damage lumbar vessels. There is great variability of vascular anatomy in front of L5-S1 disc space. The left common iliac vein is at greater risk than the common iliac arteries [3]. Ebraheim et al. found a triangular safety zone averaging $60 \mathrm{~mm}$ in width and $40 \mathrm{~mm}$ in height between the left common iliac vein and the right common iliac artery in 40 human cadavers [17]. In our study, we found that the distance from the abdominal aortic bifurcation to the L5 lower edge was $40.50 \pm$ $9.40 \mathrm{~mm}$, the distance from the common iliac vein confluence to the L5 lower edge was $27.80 \pm 8.60 \mathrm{~mm}$, and the horizontal distance from the inner edge of the common iliac vein to the L5 lower edge was 37.50 $\pm 1.30 \mathrm{~mm}$, and the safe operating area was $2058.20 \pm 84.30 \mathrm{~mm}^{2}$.

Lumbar fusion has been shown to accelerate adjacent segments degeneration (ASD). It was reported that ALIF may have an advantage over PLIF in preventing the developing ASD [18]. Compared with PLIF, ALIF reduced the damage to the integrity of the posterior complex, which may be helpful in preventing accelerated adjacent segment degeneration after spinal fusion [19]. Although percutaneous PPS was applied during ALIF procedure in these studies [20], we could believe that APS had comparable results to PPS with the potential benefit of preventing accelerated ASD, and more studies should be performed on this topic.

\section{Conclusions}

We successfully measured the projection, screw trajectory angle, and BSPL of the anterior transpedicle screw in L5 and S1, proved its insertion regularity, and determined the safe operating area. These results can guide APS insertion and improve its accuracy. We plan to use the APS in L5 and S1 based on these results and will evaluate the accuracy of the positioning of the screw.

\section{Abbreviations}

ALIF anterior lumbar interbody fusion

APS anterior pedicle screw 
BSPL bone screw passageway length

PLIF posterior lumbar interbody fusion

PPS posterior pedicle screw

\section{Declarations}

\section{Ethics approval and consent to participate}

This study was approved by the Ethics Committee of Tongde Hospital of Zhejiang Province, and the methods were carried out in accordance with the approved guidelines.

\section{Consent for publication}

Not applicable

\section{Availability of data and materials}

The datasets used and/or analysed during the current study are available from the corresponding author on reasonable request.

\section{Competing interests}

The authors declare that they have no conflicts of interest.

\section{Funding}

The study was supported by the Zhejiang Province Public Welfare Technology Application Research Project (CN) (LGF19H060006). The costs involving designing the study, collection, analysis, interpretation of data are funded by this project.

\section{Authors' contributions}

XW: project development, manuscript writing, funding acquisition. XB: data collection, data analysis, manuscript writing. DW: data analysis, manuscript writing. SH: project development, data collection. LD: data analysis, supervision. HT: data collection, supervision. All authors have read and approved the manuscript.

\section{Acknowledgment}

We thank Nancy Schatken BS, MT(ASCP), from Liwen Bianji, Edanz Group China (www.liwenbianji.cn/ac), for editing the English text of a draft of this manuscript.

\section{References}


1. Giang G, Mobbs R, Phan S, et al. Evaluating outcomes of stand-alone anterior lumbar interbody fusion: a systematic review. World Neurosurgery. 2017; 104: 259-271.

2. Hoff E K, Strube P, Pumberger M, et al. ALIF and total disc replacement versus 2-level circumferential fusion with TLIF: a prospective, randomized, clinical and radiological trial. European Spine Journal. 2015; 24(10):1-3.

3. Karim A, Mukherjee D, Ankem M, et al. Augmentation of anterior lumbar interbody fusion with anterior pedicle screw fixation: demonstration of novel constructs and evaluation of biomechanical stability in cadaveric specimens. Neurosurgery. 2006; 58(3):522-527.

4. Choi KC, Kim JS, Shim HK, et al. Changes in the Adjacent Segment 10 Years After Anterior Lumbar Interbody Fusion for Low-Grade Isthmic Spondylolisthesis. Clinical Orthopaedics \& Related Research. 2014; 472(6):1845.

5. Wang WJ, Chen WK, Yan YG, et al. Application of anterior debridement and reconstruction with anatomical screw-plate fixation for lumbosacral tuberculosis: A 2-year-plus follow-up. Medicine. 2017; 96(26):e7103.

6. Wang W, Liu S, He G, et al. Application of Laparoscopic Lumbar Discectomy and Artificial Disc Replacement: At Least Two Years of Follow-Up. Spine. 2016; 41 Suppl 19(19):B38.

7. Inamasu J, Kim DH, Logan L. Three-dimensional computed tomographic anatomy of the abdominal great vessels pertinent to L4-L5 anterior lumbar interbody fusion. Minim Invasive Neurosurg. 2005; 48(03):127-131.

8. Ebraheim NA, Rollins JR Jr, Xu R, et al. Projection of the lumbar pedicle and its morphometric analysis. Spine (Phila Pa 1976). 1996; 21(11):1296-300.

9. Boucher HH. A method of spinal fusion. Journal of Bone \& Joint Surgery-british Volume. 1959; 41(2):248-259.

10. Kasten MD, Rao LA, Priest B. Long-term results of iliac wing fixation below extensive fusions in ambulatory adult patients with spinal disorders. Journal of Spinal Disorders \& Techniques. 2010; 23(7):37-42.

11. Wang $G$, Yang $H$, Chen $X$, et al. Standard fluoroscopic views in cadavers for determining the entry point and depth of a guide needle for use in transpedicular procedures of the thoracolumbar spine. Journal of Clinical Neuroscience. 2010; 17(5):588-591.

12. Bai JY, Zhang W, An JL, et al. True anteroposterior view pedicle screw insertion technique. Therapeutics \& Clinical Risk Management. 2016; 12(Issue 1):1039-1047.

13. Choi KC, Kim JS, Shim HK, et al. Changes in the Adjacent Segment 10 Years After Anterior Lumbar Interbody Fusion for Low-Grade Isthmic Spondylolisthesis. Clinical Orthopaedics \& Related Research. 2014; 472(6):1845.

14. Weinstein JN, Spratt KF, Spengler D, et al. Spinal pedicle fixation: reliability and validity of roentgenogram-based assessment and surgical factors on successful screw placement. Spine. 1998; 13(9):1012. 
15. Xin DU, Zhao LX, Qi bin YE. Radiological anatomy study in selecting the length of pedicle screw in lumbar spine. Chinese Journal of Clinical Anatomy. 2002; 20:15-7. [in Chinese]

16. Acikbas SC, Tuncer MR. New method for intraoperative determination of proper screw insertion or screw malposition. J Neurosurg. 2000; 93(1 Suppl): 40-4.

17. Ebraheim NA, Xu R, Farooq A, et al. The quantitative anatomy of the iliac vessels and their relation to anterior lumbosacral approach. J Spinal Disord, 1996; 9:414-417.

18. Lee DY, Lee SH, Maeng DH. Two-level anterior lumbar interbody fusion with percutaneous pedicle screw fixation: a minimum 3-year follow-up study. Neurologia medico-chirurgica, 2010; 50(8):645.

19. Park P, Garton HJ, Gala VC, et al. Adjacent segment disease after lumbar or lumbosacral fusion: review of the literature. Spine, 2004; 29(17):1938-1944.

20. Min JH, Jang JS, Lee SH. Comparison of anterior- and posterior-approach instrumented lumbar interbody fusion for spondylolisthesis. J Neurosurg Spine, 2007; 7(1):21-26.

\section{Tables}

Table 1. Radiographic parameters of anterior entered point, screw angle and bone screw passageway length in L5 and S1 $(x \pm s, n=40)$

\begin{tabular}{lcc}
\hline & L5 & S1 \\
\hline Distance to upper endplate $(\mathrm{mm})$ & $12.5 \pm 1.3$ & $4.54 \pm 0.87$ \\
Distance to lower endplate $(\mathrm{mm})$ & $17.3 \pm 1.6$ & - \\
Distance to midline $(\mathrm{mm})$ & $6.6 \pm 0.7$ & $6.6 \pm 0.6$ \\
Transverse screw angle $\left({ }^{\circ}\right)$ & $25.3 \pm 2.8$ & $25.7 \pm 2.6$ \\
Sagittal screw angle $\left({ }^{\circ}\right)$ & $17.1 \pm 1.7$ & $22.4 \pm 1.1$ \\
Bone screw passageway length $(\mathrm{mm})$ & $48.6 \pm 3.5$ & $48.0 \pm 3.5$ \\
\hline
\end{tabular}

\section{Figures}




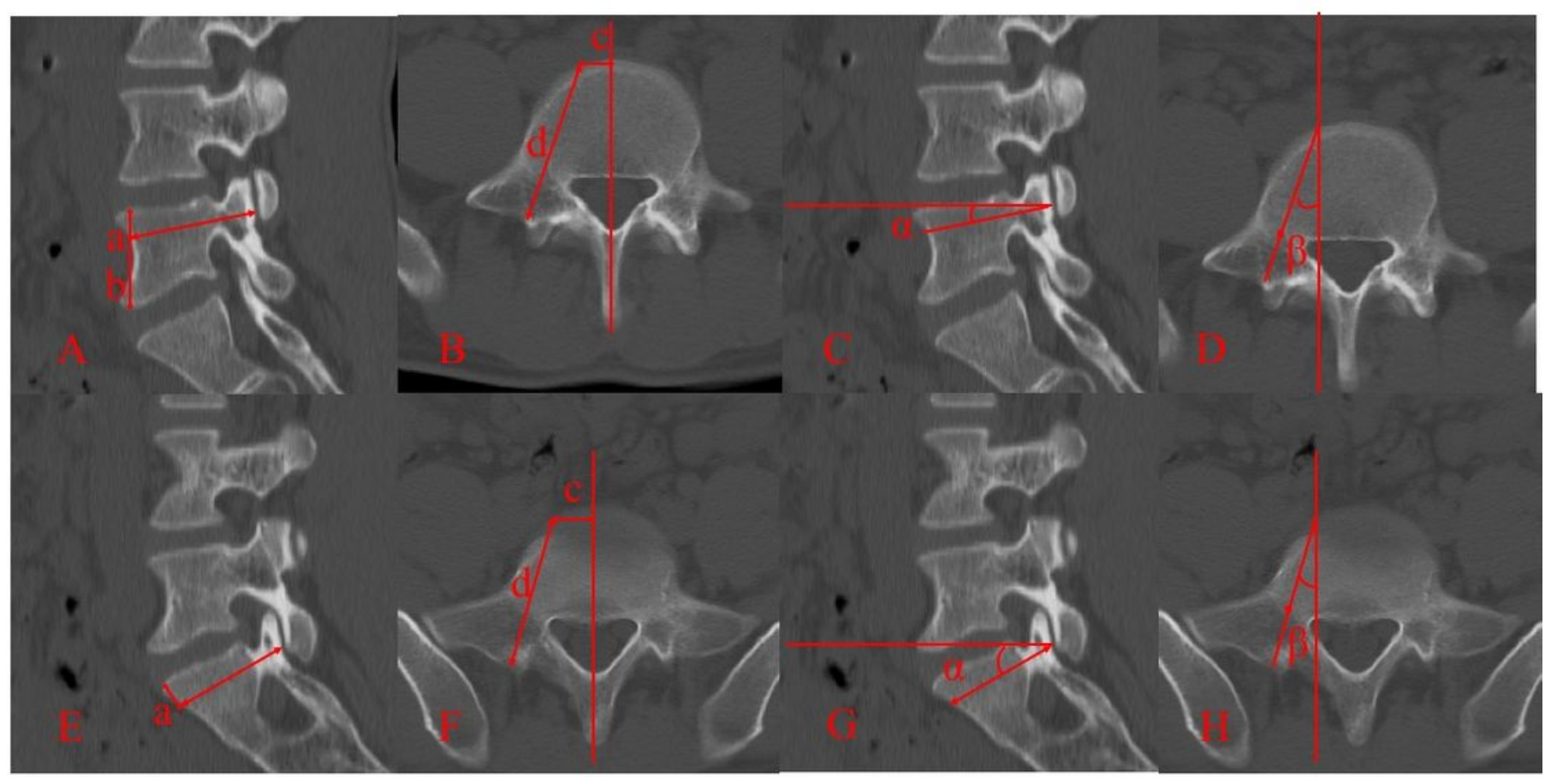

\section{Figure 1}

Radiographic parameter measurements were performed, including: anatomic parameters of anterior projection (the distances to upper endplate, lower endplate and midline), transverse screw angle, sagittal screw angle and BSPL. 


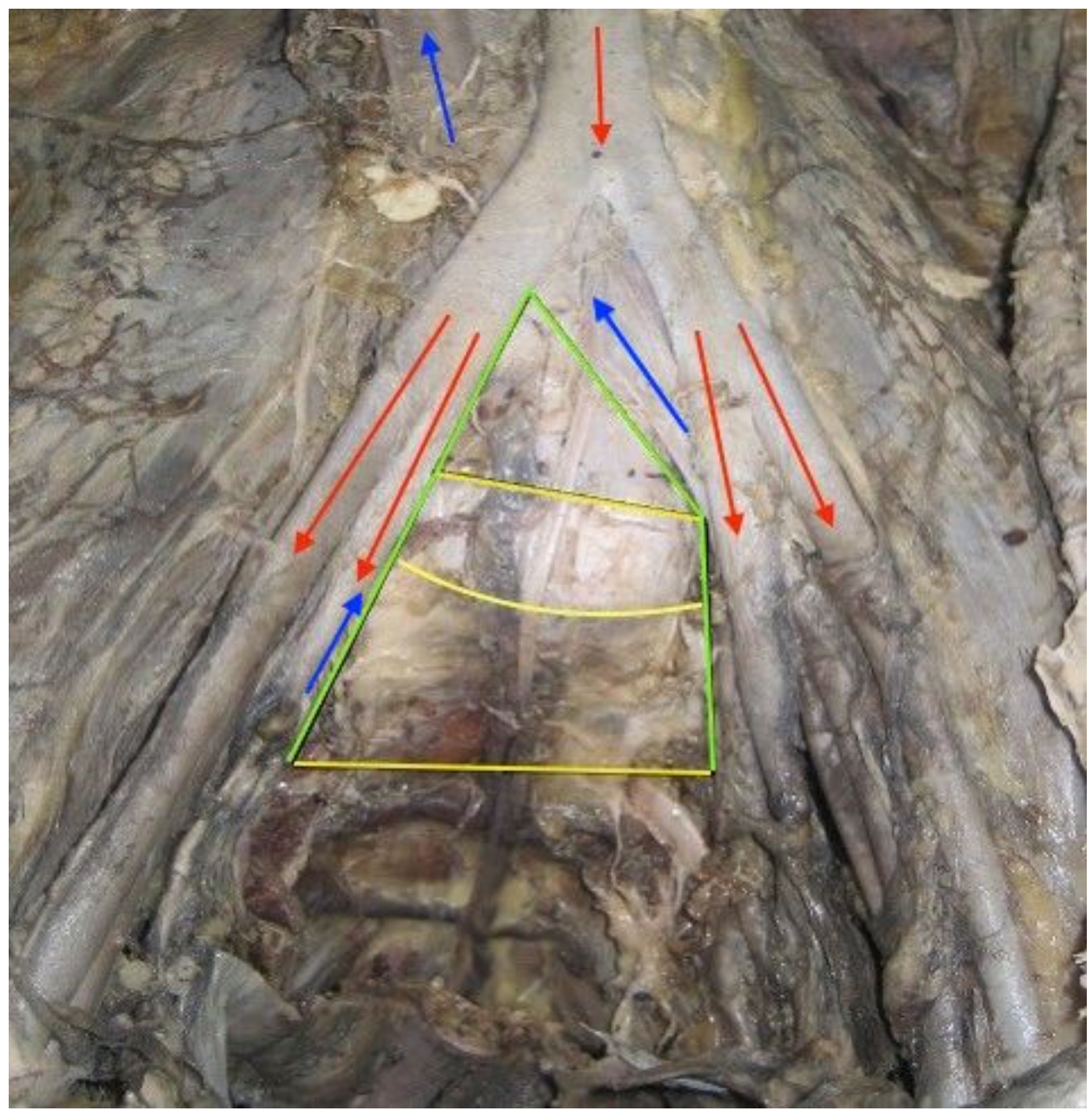

\section{Figure 2}

The safe operating area. The red arrows represent arteries; the blue arrows represent veins; the green lines represent the inner edge of internal iliac veins; the upper yellow line represents the lower endplate of L5; the middle yellow line represents the upper endplate of $\mathrm{S} 1$; the lower yellow line represent the connecting line between the both side sacral foramens of S1. The safe operating area is the within the area of the green lines and the lower yellow line. 


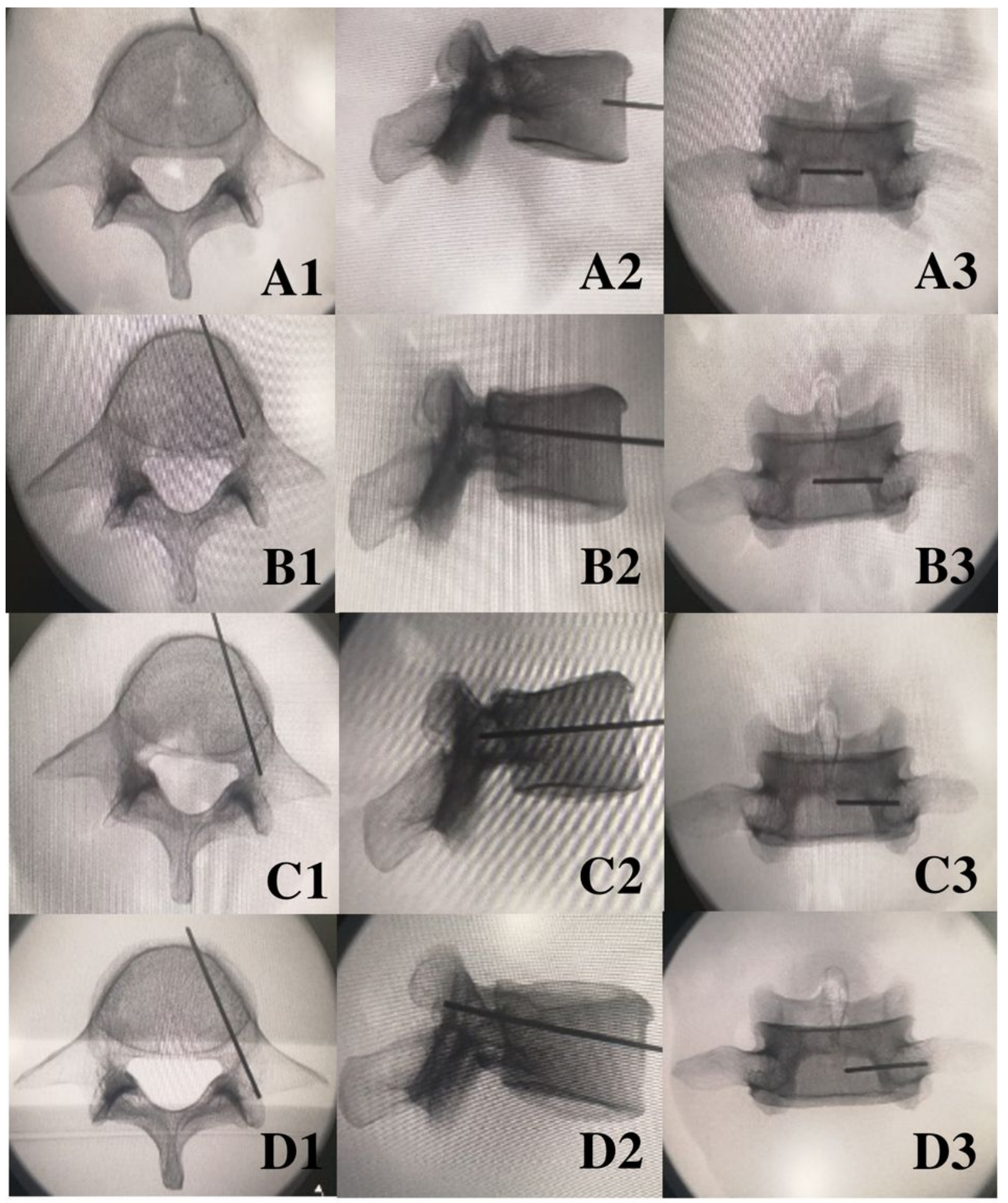

Figure 3

The regularity of $L 5$ anterior pedicle screw insertion. A2: anterior projection point in the lateral view; A3: anterior projection point in the AP view; B2: middle of the vertebrae in the lateral view; B3: middle of the vertebrae in the AP view; $C 2$ : posterior edge of the vertebrae in the lateral view; C3: posterior edge of the vertebrae in the AP view; D2: posterior projection point in the lateral view; D3: posterior projection point in the AP view. 


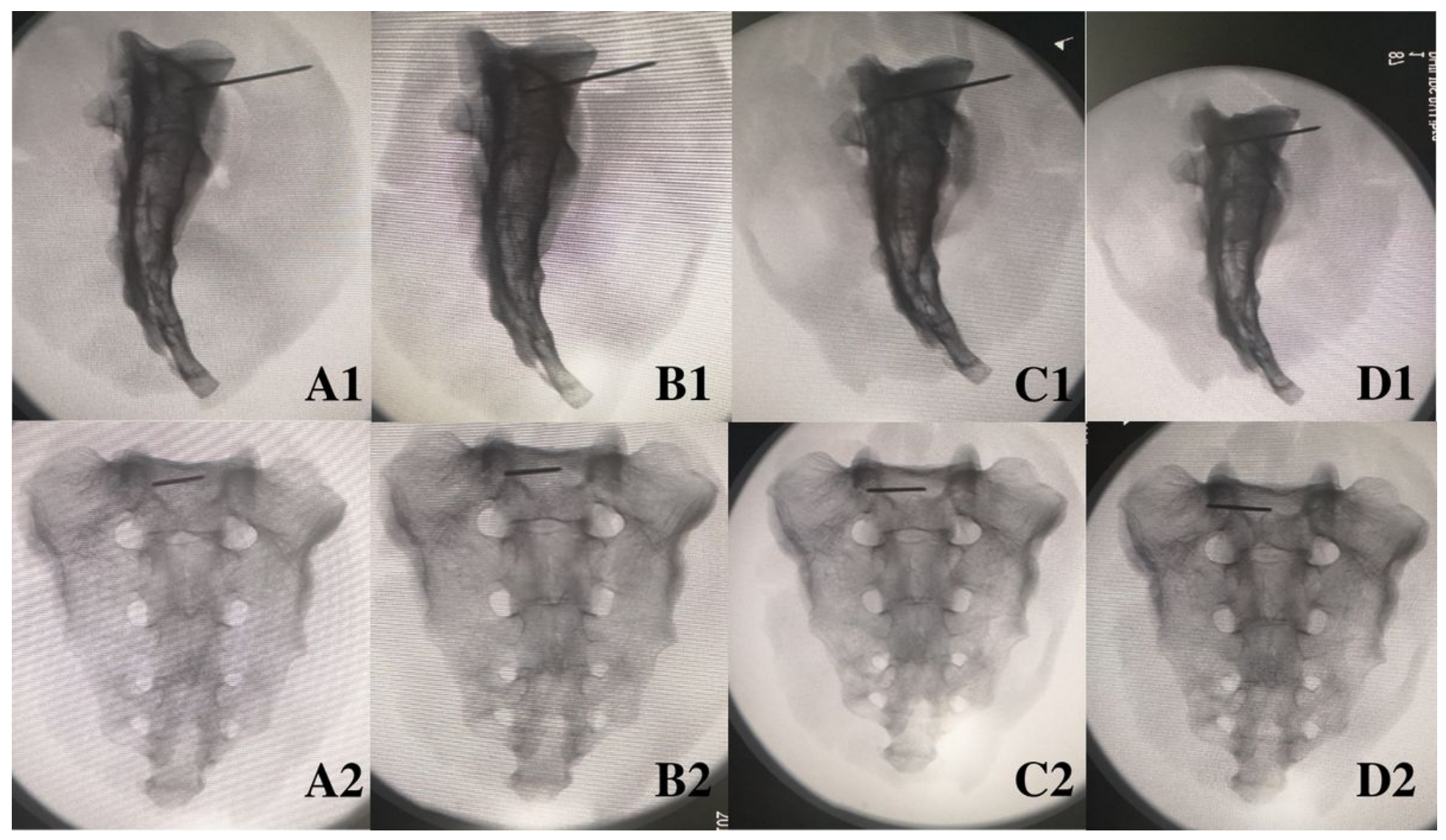

Figure 4

The regularity of $\mathrm{S} 1$ anterior pedicle screw insertion. A1: anterior projection point in the lateral view; $\mathrm{A} 2$ : anterior projection point in the AP view; B1: middle of the vertebrae in the lateral view; B2: middle of the vertebrae in the AP view; $\mathrm{C} 1$ : posterior edge of the vertebrae in the lateral view; $\mathrm{C} 2$ : posterior edge of the vertebrae in the AP view; D1: posterior projection point in the lateral view; D2: posterior projection point in the AP view. 